\title{
Steering in Video-Based Driving Simulation with Stereo Depth Maps Dynamic Perspective Corrections
}

\author{
Michael Brogan, Charles Markham, Sean Commins, and Catherine Deegan
}

\begin{abstract}
This paper describes a video-based driving simulator that allows the user to steer through a video sequence acquired by a mobile mapping system. This mapping system acquired geotagged stereo video at a rate of $10 \mathrm{~Hz}$. The video data were collected from a standard vehicle, which was driven along a road under normal road conditions. With the use of the stereo image data, a method that allowed a participant to steer through a video was implemented. An experiment to assess a driver's response to this steering is presented. Drivers were found to respond to the geometry of the video sequence by steering left or right when expected.
\end{abstract}

The importance of a steering component being introduced to any video-based driving simulator is clear, as steering is one of the main control mechanisms of driving (1). The absence of a steering component in a driving simulator would reduce the usefulness of such a tool significantly. Research that has used driving simulators has covered many different areas, for example, medical studies, driver distraction, steering behavior, and driver response times (2-7). Speed is recognized as a factor in road traffic accidents, and is linked to a driver's perceived ability to negotiate road geometry $(8,9)$. A driver's failure to control steering at higher speeds introduces a significantly increased risk. Although in some instances increased speed may not be inherently dangerous, the decreased ability of a driver to control steering at the increased speed is.

As technological advances make available ever-increasing processing power at ever-decreasing costs, the ability to create realistic and detailed graphical worlds for driving simulation has been the subject of much research (10). Even outside the simulator community, consumer grade graphical devices have the ability to provide the user with visual streams that offer never-before-seen levels of photorealism, for example, the Microsoft Xbox One, and the Sony PlayStation $4(11,12)$. However, despite these advances, the devices remain model-based. Although termed photorealistic, they are not truly such. Whether the discrepancy observed in these environments occurs because of subtle differences in modeling environmental parameters, such as lighting and shadowing, or, perhaps, a temporary progression into the uncanny valley associated with the boundaries of graphical realism, is unclear (13).

M. Brogan and C. Deegan, Department of Engineering, Institute of Technology Blanchardstown, Blanchardstown Road North, Dublin 15, Ireland. C. Markham, Department of Computer Science, and S. Commins, Department of Psychology, National University of Ireland, Maynooth, County Kildare, Ireland. Corresponding author: M. Brogan, michael.brogan@itb.ie.

Transportation Research Record: Journal of the Transportation Research Board, No. 2518, Transportation Research Board, Washington, D.C., 2015, pp. 104-112. DOI: 10.3141/2518-14
The increase in technological processing power has also led to the development of relatively low-cost imaging devices that, among other benefits, offer pixel-locked, synchronized stereo imagery and can be interfaced directly with external triggering devices. The increased availability of such cameras has led to several video-based driving simulators (14-18). Although some of these simulators have replaced the graphical textures of model-based simulators with photographic textures, others have allowed for a switch between dual feeds of videos acquired in two lanes of a motorway (16). Nevertheless, none of these video-based simulators allows for a true gradient steering effect when steering through videos.

The capacity to introduce a realistic form of steering control in a video-based driving simulator is important because measurement of driver position requires the ability of participants to steer in a realistic fashion and because speed choice influences steering control.

Having one simulation of steering or one simulation of speed without the other, outside validation studies, reduces the realism of the experience and the validity of the data. Research has suggested that the highest form of validation is one in which there is a comparison of simulation data with real-world data (19). There must therefore be two comparable scenarios available, one from an on-the-road driver, and another from a simulator driver. Direct comparison between the two scenarios is difficult, as measurable properties between the two are complex to synchronize and correlate. Acquisition of data describing a driver's speed and position is a trivial issue once the vehicle has been equipped with a time-stamped GPS module that exhibits a reasonable level of accuracy. Similarly, for graphicsbased simulators, the virtual world provides unparalleled levels of control of the driver's environment. However, synchronization between the real world and the virtual world requires the generation of the graphical environment with known properties of the real-world environment.

Even if accurate modeling is achieved, simulation adaptation patterns have been noted (20). With the generation of the graphical environment, absolute synchronization between the real world and the virtual world may remains an issue. GPS accuracy or availability, actual driver trajectory, and traffic on the road at the time of data acquisition that is absent from the graphical model may affect direct comparisons between the real world and the virtual world (21). Traffic on the real road may affect the driver's speed, which results in an additional parameter that must be introduced to the model.

Success has been shown in introducing a steering component (22), in which a single video sequence along a straight road was adapted for use in a driving simulator. By texturing an estimated intersecting plane descriptor, a user could change lanes. This study showed that 
$87 \%$ of 220 lane change requests were made with this method, with the remaining $13 \%$ being attributed to lack of participant concentration on the change lane indicator. This experiment demonstrated that participants experienced a lane change effect following the steering action they were requested to take. The absence of stereo video data means that this approach can only introduce a sense of depth on the road plane, while other features, such as road signs, remain static in terms of perspective corrections. At present, this technique can only be applied to straight roads. The technique described in this paper allows a driver to steer around corners in a prerecorded video sequence.

\section{OUTLINE OF PRINCIPAL FINDING}

The method described in this paper uses data collected by a lowprofile mobile mapping system, consisting of stereo images and high-accuracy positioning data acquired at a rate of $10 \mathrm{~Hz}$. The positioning data are used for two purposes. The first purpose is to equalize the video for speed because it was acquired at non-constant road speed. This equalization process produces a video that plays back as though captured at a constant speed of $72 \mathrm{~km} / \mathrm{h}$ (44.7 mph). The second purpose is to establish a ground-truth data set; the positional data acquired were time-stamped, which allows for the speed of the driver in the real car on the real road to be logged. Information regarding the depth of features from the mapping system camera was derived from the stereo image data and then used to introduce a steering element to the prerecorded video sequence.

An experiment was undertaken in which the speed of the driving simulator participants was compared with the ground-truth speed of the mobile mapping system, and the steering response of the participants was compared with a training data set, which was generated without the use of the mapping system data.

\section{FRAMEWORK}

This paper describes the method by which a steerable video (i.e., a video with which a participant has the ability to change the viewing perspective) was integrated with a driving simulator. The driving simulator is described, followed by a description of the methods used to generate the video's depth description sequence. The introduction of steerable video is then detailed. An experiment that examines driver response to the steerable video in driving simulation is described, and the results of this experiment are then presented. Conclusions on these results are then discussed. The paper concludes with a discussion on future work with the video-based driving simulator.

\section{MATERIALS, METHODS, AND DATA}

\section{Driving Simulator Cabin and Control System}

The work described in this paper was built on previous research with a nonsteerable video, whose GPS-based geotags were used to generate a synchronized, sparsely populated graphical model. That previous research validated the use of video as a tool with which to measure driver speed in driving simulation when compared with the use of graphical models and real driving (18).
The driving simulator used for the purposes of testing the methods described in this paper consisted of a Microsoft Windows 7-based PC with solid-state drive and a Logitech G27 gaming steering wheel and pedals. The solid-state drive was used to increase playback speed, thereby reducing steering response time to a realistic period. These devices were used to run the simulator software and allow for user control over the simulator environment (23). A single $58 \mathrm{~cm}$ (23 in.) $1,920 \times 1,080$ resolution high-definition monitor was used for display purposes.

\section{Data Acquisition System and Route Selection}

The route chosen for data acquisition was the $\mathrm{R} 156$ regional road in County Meath, Ireland. This is a subsection of the $17.4 \mathrm{~km}(10.8 \mathrm{mi})$ Summerhill to Dunboyne route (18). This road was chosen because it offers a high level of sinuosity, along with a range of speed limit sections from $50 \mathrm{~km} / \mathrm{h}(31 \mathrm{mph})$ to $80 \mathrm{~km} / \mathrm{h}(50 \mathrm{mph})$. The route described in the video is approximately $7 \mathrm{~km}(4.35 \mathrm{mi})$ in length. The mobile mapping system data acquisition system features a self-contained stereo camera and high-accuracy GPS receiver with less than $5 \mathrm{~cm}$ ( 2 in.) positional error. The camera and navigational sensor are interfaced to allow for the logging of positional and timing data with synchronized stereo image data at a rate of $10 \mathrm{~Hz}(24-26)$.

\section{Previous Research Undertaken with Video-Based Driving Simulation}

An on-the-rails video sequence was used previously in the driving simulator to validate video as a tool with which to measure driver speed responses when compared with the use of a graphical model and a driver on a real road. Strong correlations between the model, on-therails video, and ground-truth speeds were observed, with differences attributed to the time taken for the driver of the real car to change gears in a manual gearbox and to the calibration of the acquisition vehicle's speedometer (18).

\section{Equalization of Video Speed}

To remove the acceleration and deceleration effects present in the original video, an evenly-spaced route was generated on the basis of the positional data acquired by the mapping system. A nearest-neighbor Euclidean distance linear search was performed; this procedure allows the frame nearest to each of the evenly spaced coordinates to be found. The redundant frames were then dropped from the video; this procedure allows the processed video to play back as though recorded at a constant speed of $72 \mathrm{~km} / \mathrm{h}$ (44.7 mph). An example of this is shown in Figure 1.

This equalization method differs from the previous equalization routine, as the equalized video is generated before introduction to the driving simulator, as opposed to the original approach, which used a look-up table during video playback (18).

This equalization of video playback speed also allowed for a robust method of determining the correct delay to introduce to the video sequence dependent on accelerator pressure. As the video was equalized to $72 \mathrm{~km} / \mathrm{h}(44.7 \mathrm{mph})$ at 10 frames per second, the correct delay can be achieved by changing the number of frames per second, for example, at half this speed, $36 \mathrm{~km} / \mathrm{h}$ (22.4 mph), the frame rate must be halved to five frames per second. 


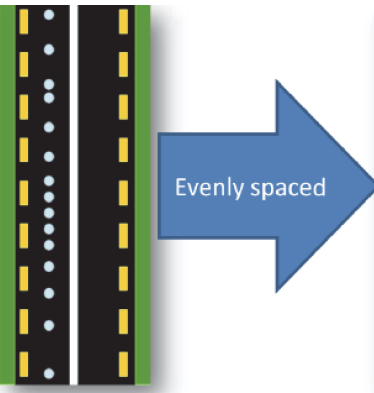

(a)

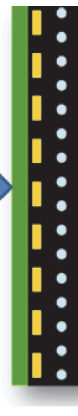

(b)

FIGURE 1 Equalization of video playback speed: (a) original trajectory of vehicle, (b) trajectory after positional data have been evenly spaced, and (c) nearest image to evenly spaced coordinate being selected.

\section{Steerable Video}

Stereo video refers to two video sequences, in which each pair of corresponding frames is acquired at the same time, to allow viewing the same scene from two differing vantage points. When acquiring stereo images from a moving platform, any delay between the acquisitions of the two introduces a source of error. Synchronization of the stereo cameras therefore simplifies the generation of the stereo depth map. For example, a $5 \mathrm{~ms}$ delay when traveling at $80 \mathrm{~km} / \mathrm{h}$ (50 mph) represents a discrepancy of more than $11 \mathrm{~cm}$ (4.3 in.) between the two images.

Once a stereo video has been acquired, each set of corresponding stereo frames can be processed to produce a single grayscale image that describes the depth of each feature visible in both stereo images. The generation of these depth maps is a three-step process involving the recovery of specific camera properties (camera calibration), correction of image distortions (undistortion and rectification), and feature matching across the two images (disparity recovery through correspondence) (27-29). These steps were implemented with the OpenCV image processing library, and are described briefly in the following sections (30).

\section{Camera Calibration}

Camera calibration is the term given to the recovery of the specific physical properties of a camera, and can be performed by acquiring a sequence of images of a checkerboard pattern at varying orientations. The imaged points are related to the physical dimensions of the checkerboard pattern, and can be processed with linear algebra to recover the camera's physical properties. Figure $2 a$ shows an example of the checkerboard.

These properties are divided into the intrinsic parameters (the lens focal length and principal point) and the extrinsic parameters (the rotation and translation of the camera relative to some arbitrary external coordinate system). Distortions introduced by the camera lens, while not strictly internal to the camera, are usually considered as part of the intrinsic parameter recovery process. Although the extrinsic parameters are calculated relative to an arbitrary external coordinate system, as defined by the checkerboard, when dealing with stereo cameras, the prime importance is the recovery of the rotation and translation of the two cameras relative to each other. Independent calibration of two cameras can recover this relative transformation once the same calibration images are used for both.
Successful stereo calibration therefore returns three sets of required data: the intrinsic parameters of each camera, the rotation and translation of the two cameras relative to each other, and the lens distortion coefficients of each camera. With these data, generation of the grayscale depth maps becomes possible.

\section{Undistortion and Rectification}

Distortion by a camera lens occurs primarily on the image periphery and is termed radial lens distortion (RLD). Two variants of RLD exist: pin cushion and barrel. The former causes straight lines on the image periphery to curve inward toward the center of the image, and the latter causes these lines to curve outward away from the center of the image. In both cases, RLD is described by a series of coefficients. Once these are recovered, the original image can be corrected, essentially presenting an image as if it were acquired through a perfect lens.

The extrinsic parameters can then be applied to the two images, reorienting each such that corresponding features lie on the same horizontal image coordinate (i.e., $y$-coordinate) in both images of the stereo image pair, essentially presenting stereo images as though they were acquired by front-parallel (facing in exactly the same direction) stereo cameras. These undistortion and rectification steps are used to prepare and simplify the next process.

\section{Disparity Recovery}

Once they are undistorted and rectified, the corresponding features are then matched between the left and right images. As rectification has already been performed, the correspondence matching becomes a one-dimensional matching process as opposed to the twodimensional matching process that would otherwise be required. This process allows a one-dimensional disparity value of each feature to be recovered and, as disparity can be considered a function of depth, the disparity values are used to generate a depth map description of the scene acquired by the stereo images.

\section{Depth Maps}

The grayscale descriptor returned by the disparity measurement function is termed a depth map, and assigns a grayscale value to each 


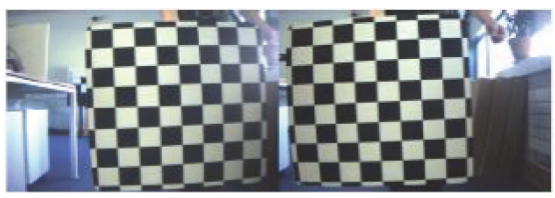

(a)

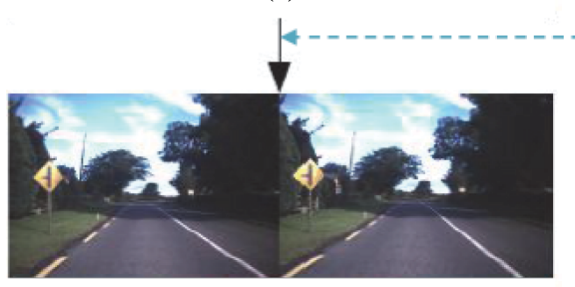

(b)

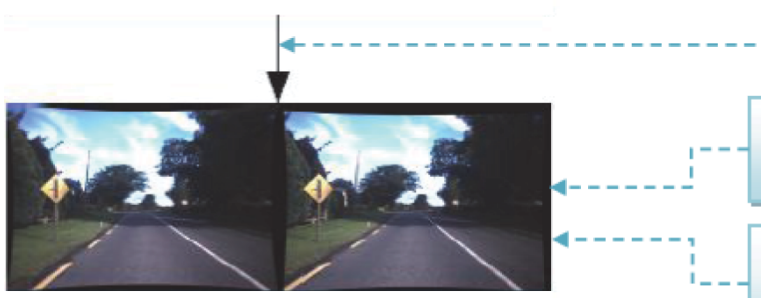

(c)

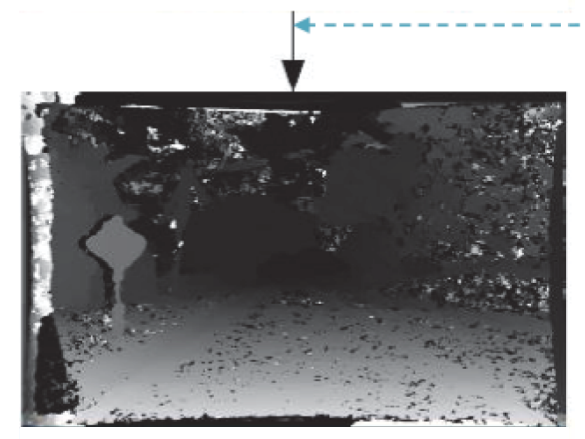

(d)

Use these images to measure disparity and generate depth maps

FIGURE 2 Generation of depth maps from stereo images: (a) calibration images, $(b)$ original distorted images, $(c)$ undistorted and rectified images, and $(d)$ resultant grayscale depth map.

feature corresponding to the depth of that feature from the reference camera. These values are typically in the 8-bit range, allowing for a resolution of 256 distances. A zero grayscale value represents a feature far from the camera, and a 255 grayscale value represents a feature very close to the camera. An example of each stage of the depth map generation process is shown in Figure 2.

\section{Steerable Video}

Texturing the depth map with its corresponding video frame can be used to generate a three-dimensional environment, thereby allowing steering to be introduced into a video-based driving simulator. To achieve this environment, the intensity of each depth map pixel is used to assign the distance at which the corresponding video frame color pixel will be located. This environment was developed with the OpenCV image processing and FreeGLUT graphical libraries. An example is shown in Figure $3(30,31)$.

The virtual camera of the driving simulator environment is focused on the central horizon point, allowing the position of the driver to

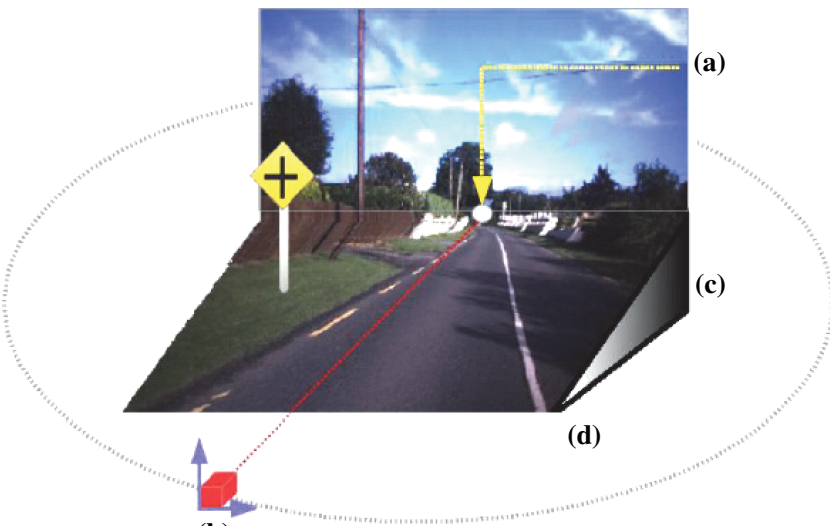

(b)

FIGURE 3 Generated video-based virtual coordinate system: (a) center of focus of virtual camera, (b) camera location, and $(c)$ to $(d)$ gradient that indicates relationship between feature's depth from camera relative to corresponding depth map intensity value. 
change relative to the position of the driving simulator's steering wheel. When the relative position is the same as that of the original acquisition video, the original video frame will be displayed, and when the relative position is changed, a video frame will be transformed to reflect the change in position.

Lateral placement is represented in a limited fashion by the use of the virtual arcball camera. A constant steering angle will change the perspective for successive frames. If the steering angle deviates significantly from the actual angle required, a lack of sufficient texturing will be observed in data absent from the original video frame. Lateral roadway translation is represented by the camera moving around a point on the image line representing the horizon. The camera orbits this horizon point, meaning that a constant translation will circle the scene plane. The effect of this translation was limited such that the camera can only orbit the front of this plane, that is, within a range of \pm 90 degrees. In practice, the redundancies introduced by the lack of image data become excessive within approximately \pm 30 degrees, as the camera used has a 66-degree field of view. Use of other camera lens types, for example, a fish-eye camera lens, could increase this range.

\section{Correction of Simulator's Steering Wheel Position}

The simulator's steering wheel position at each textured frame must reflect the position of the vehicle at the instance of data acquisition. For example, if the data acquisition vehicle's steering wheel was oriented at 45 degrees when the vehicle enters a bend in the road, the steerable video environment must reflect this. Neglecting to perform this step would require the simulator's steering wheel to remain in a neutral position to navigate the steerable video along the route. A steering wheel ratio of 12:1 was chosen; that is, for each 1-degree turn in the vehicle wheels, a 12-degree turn in the steering wheel was required.

A training set was used to generate an estimate of the correct steering wheel position given the geometry of the road. Once the training set data were applied to the control system, a driver would have to control the steering to ensure the car remained on the road. An alternative method that uses the steering position of the mapping system vehicle, derived directly from GPS data, is being investigated but was not used for the data presented in this paper. The aim of this correction is to force the participant to correct the camera position to a zero lateral coordinate, allowing each video frame to be presented as originally acquired. By introducing an offset angle from the training set, the user is required to steer to force the position of the virtual camera to move to a zero lateral position.

\section{Insertion of Vehicle Windshield and Speedometer}

A second texture is introduced to allow a dashboard and speedometer to be displayed on the screen. The texture remains in a fixed position, while the perspective of the external environment is changed. The current speed is written to the speedometer, based on the pressure applied to the accelerator pedal. An example of the windshield and speedometer is shown in Figure 4. Various rotations and translations around a scene are shown in Figure 5.

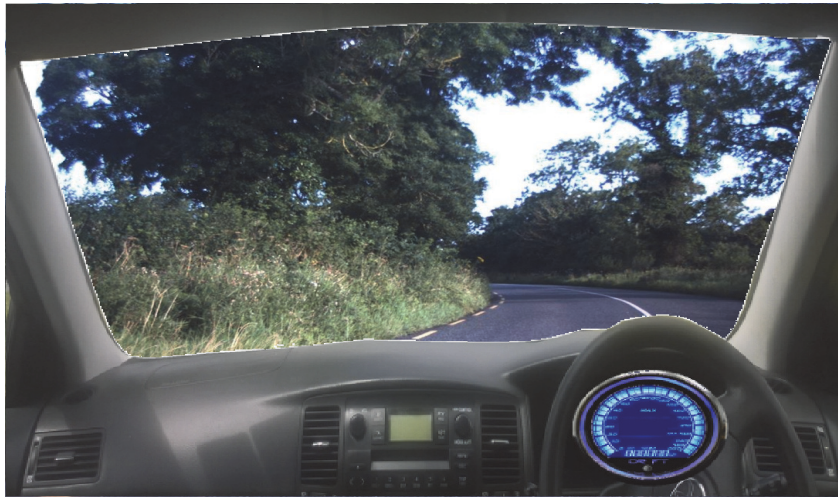

FIGURE 4 Driving simulator environment with windshield and speedometer texture.

\section{TESTING AND VALIDATION}

Eleven participants drove through the steerable video, and the driver speed and steering wheel position were collected for each participant per frame. The participants ranged in age from their early twenties to late fifties ( $\mu=34.8$ years, $\sigma=10.4)$ and consisted of eight men and three women. Before the beginning of the experiment, each participant was informed about the control system, and that the participant was to drive in a normal manner, responding to the road as if driving a real vehicle. For participants to advance through the sequence, the accelerator pedal required the application of pressure. The greater the pressure, the faster the sequence would advance. The opposite is also true; reduction in pressure would reduce the speed at which the sequence would advance. This increase and reduction in speed was related to the user in terms of the time delay between the loadings of successive video frames. The speed and vehicle position were recorded at $2 \mathrm{~m}(6.6 \mathrm{ft})$ intervals.

A running average was calculated; this calculation reduced the 3,750 data points per participant to 100 data points. The averaged participant speed and the averaged ground-truth speed are shown in Figure 6.

The angular positions of the steering wheel for each video frame for each participant were recorded. Those data represent the steering behavior of each participant on each frame. The average angular values were calculated with a mean of circular quantities calculation. A subset of the angular training set and the average angular participant data are shown in Figure 7.

\section{DISCUSSION OF RESULTS}

The results obtained from these experiments were examined for the drivers' responses to the geometry of the road in the video. For example, a left turn evidenced in the video required a simulator participant to make a similar turn with the simulator steering wheel.

Drivers were told to drive as they would normally, and that the starting segment had a posted speed limit of $80 \mathrm{~km} / \mathrm{h}(37 \mathrm{mph})$. The simulator driver steering wheel positions were consistent with road features; participants steered left, right, and straight when they were expected to do so. Driver speed was also found to be directly relevant to road features; decreases in speed were observed when encounter- 


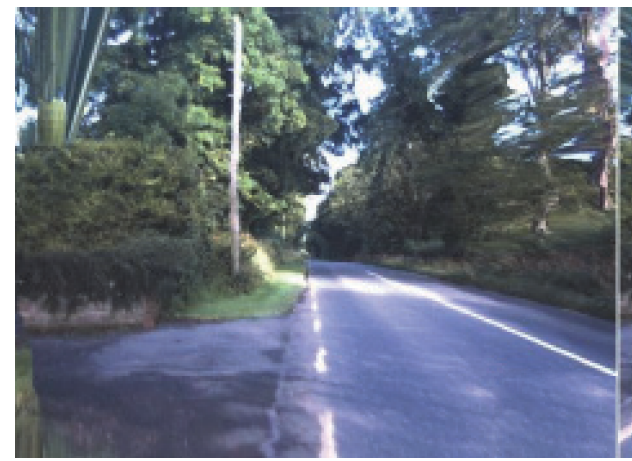

(a)

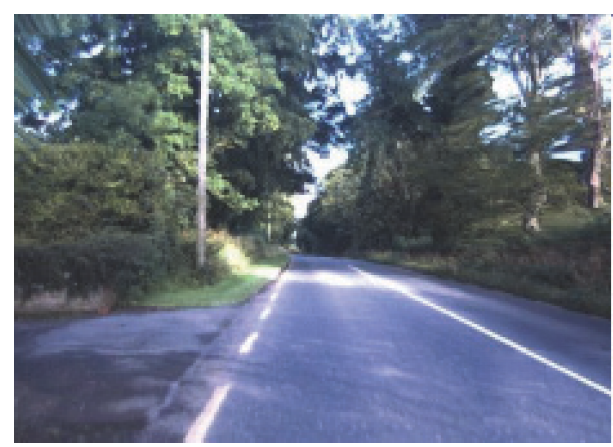

(d)

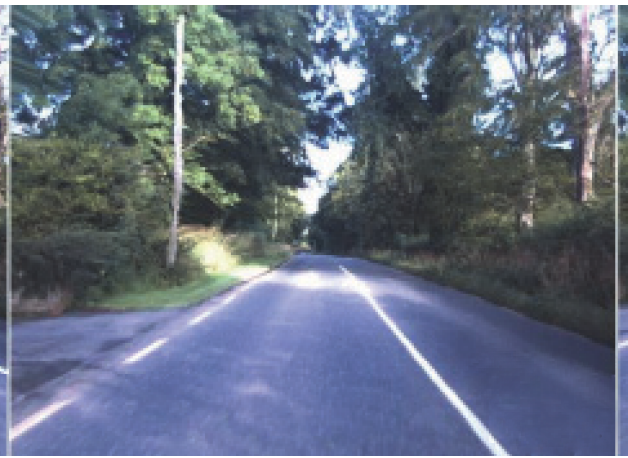

(b)

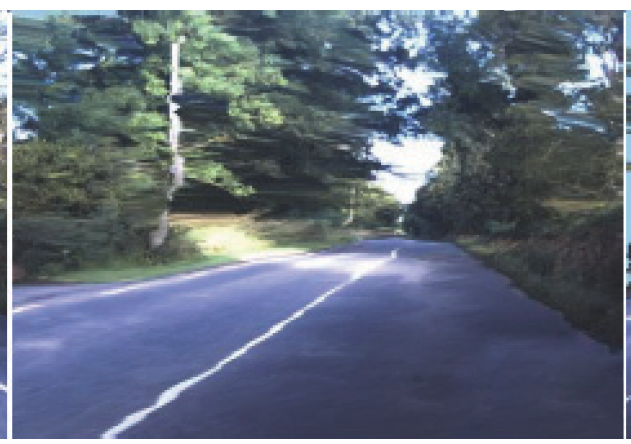

(e)

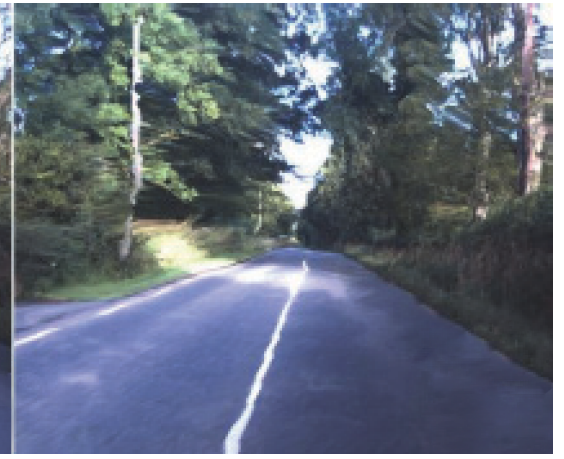

(c)

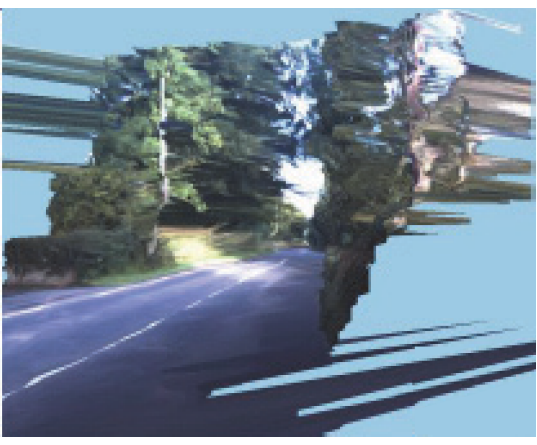

(f)

FIGURE 5 Rotations and translations around the scene: $(a)$ left rotation, $(b)$ no rotation, $(c)$ right rotation, $(d)$ left translation, $(e)$ right translation, and $(f)$ nontextured areas caused by lack of data introduced by changing perspective of scene.

ing bends in the road, and increases in speed were observed when exiting these bends.

The general form of the averaged angular signal and the training set angular signal are similar in shape; that is, participants turned left and right and drove straight where they were expected to do so. In the training set, $13 \%$ of the time was spent performing a left turn, $24 \%$ performing a right turn, and $63 \%$ performing no turn. In the aver- age simulator set, $20 \%$ of the time was spent performing a left turn, $20 \%$ performing a right turn, and $60 \%$ performing no turn. Three such sections can be seen in Figure 8, in which the angular training set requires no turn, then a left turn, and then a right turn.

A difference was observed between the speed recorded with the mapping system and the average speed recorded by the driving simulator. This difference may have been caused by several factors, such

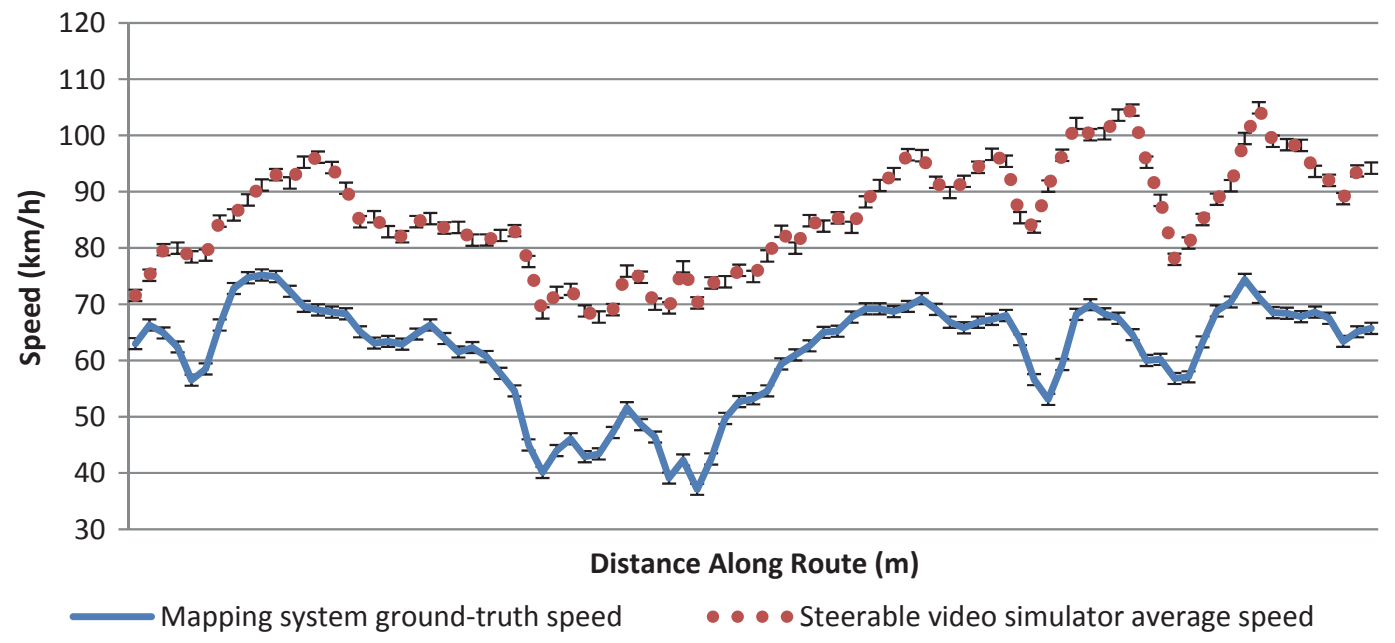

FIGURE 6 Averaged ground-truth speed acquired by the mapping system, $n=1$, and the median simulator participant speed, $n=11$ (error bars $=$ standard error). 


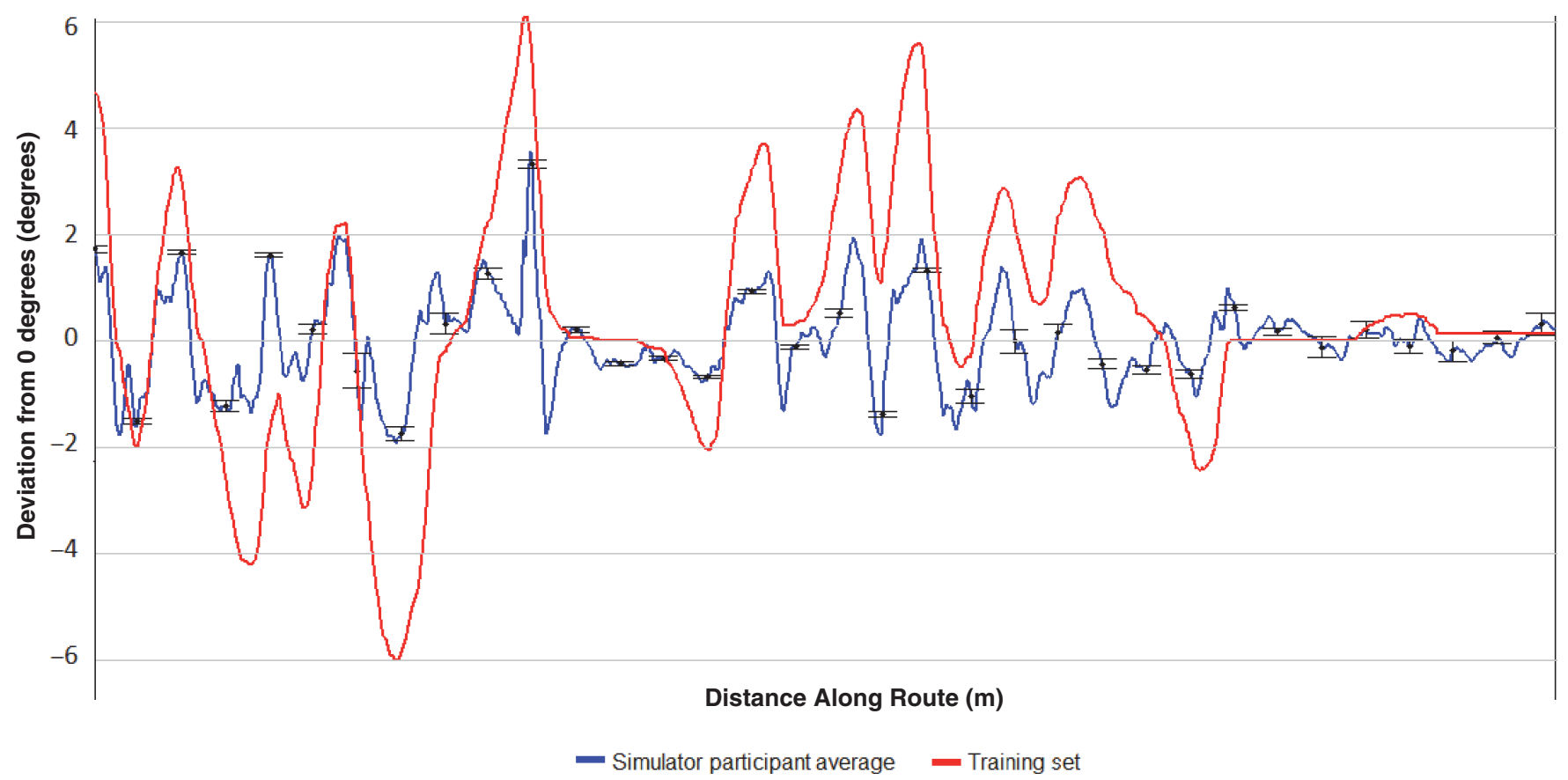

FIGURE 7 Comparison of average angle from participant steering data and training angle used to introduce required steering element to video sequence (training set angle changes location of virtual camera; this situation means that each participant needs to compensate for angle changes; error bars $=$ standard error on simulator participant average, $n=11$ ).

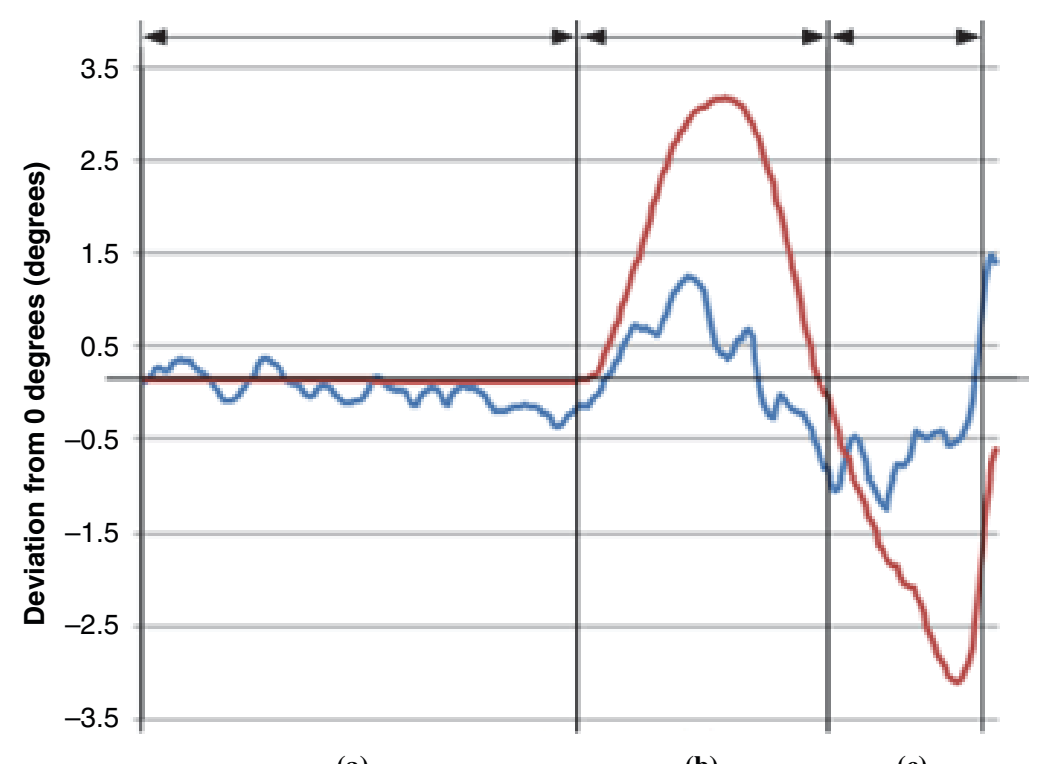

(a)

(b)

(c)

Distance Along Route (m)

- Average angle Training set angle

FIGURE 8 Examples of three steering behaviors between averaged simulator data and training data set: $(a)$ no turn, $(b)$ left turn, and $(c)$ right turn. 
as the calibration of the acquisition vehicle's speedometer. This occurred, for example, when the driver was traveling at a speedometer speed of $80 \mathrm{~km} / \mathrm{h}(50 \mathrm{mph})$, but was traveling at a true speed of $72 \mathrm{~km} / \mathrm{h}(44.7 \mathrm{mph})$. The lack of any inertial effects on the simulator participants, which were present when the ground-truth data were acquired, may also have been a contributing factor. The small sample size may also have been responsible in part for this difference.

The steering method described in Brogan et al. presented an experiment in which an explicit request to change lanes was delivered to the user via an onscreen timer (22). The experiment described in this paper requested steering behavior in a more implicit fashion, in which the steering behavior was suggested by the road geometry, rather than an externally introduced request system.

An element of understeering is observable in Figure 8, $b$ and $c$, in which the average participant's angular value is less than that of the corresponding training set angular value. This may have been caused by an element of oversteering during construction of the training set.

\section{CONCLUSIONS AND FUTURE RESEARCH}

This paper has introduced a new simulator framework that allows steerable video sequences to be introduced in driving simulation. The new framework allows a truly photorealistic environment to be generated, creating not only a high-fidelity environment, but also an environment that describes real-world routes. This environment is generated without the need for high-end graphical processing hardware and has been run on a standard Intel i5 processor-based notebook.

A present limitation of this approach concerns the lack of texturing introduced to the scene by any missing image data. For example, viewing behind a road sign will result in a blank area, as this visual information was unavailable from the images. There are several ways in which this issue may be addressed, and that will form part of future research. Other future research will include the introduction of a stereoscopic element to the driving simulator. A further aspect will be to introduce some form of head-tracking into the video sequence such that the perspective of the video will update relative to where the participant's head is facing.

Use of the mapping system's GPS data for automatic generation of the steering angle correction offset signal will allow this signal to be determined automatically. This innovation will take into account differences between the mechanics of the mapping system and the current input methods of the simulator, for example, the manual gearbox of the mapping system vehicle, and the absence of this from a simulator environment.

Research into augmentation of the steerable video with traffic is under way. Upon completion of this research and the future work, a stereoscopic steerable video with head-tracking and augmented with additional vehicles will be available.

\section{ACKNOWLEDGMENTS}

The authors thank the Irish Research Council for its continuing support and the participants for their time.

\section{REFERENCES}

1. Feenstra, P., R. van der Horst, B. J.C. Grácio, and M. Wentink. Effect of Simulator Motion Cuing on Steering Control Performance: Driving Simulator Study. In Transportation Research Record: Journal of the
Transportation Research Board, No. 2185, Transportation Research Board of the National Academies, Washington, D.C., 2010, pp. 48-54.

2. Madeley, P., J. L. Hulley, H. Wildgust, and R. H. Mindham. Parkinson's Disease and Driving Ability. Journal of Neurology, Neurosurgery and Psychiatry, Vol. 53, No. 7, 1990, pp. 580-582.

3. Rizzo, M., L. Stierman, N. Skaar, J. D. Dawson, S. W. Anderson, and S.P. Vecera. Effects of a Controlled Auditory-Verbal Distraction Task on Older Driver Vehicle Control. In Transportation Research Record: Journal of the Transportation Research Board, No. 1865, Transportation Research Board of the National Academies, Washington, D.C., 2004, pp. 1-6.

4. Tijerina, L., M. Blommer, R. Curry, J. Greenberg, D. Kockhar, C. Simonds, and D. Watson. Effects of Adaptive Lane Departure Warning System on Driver Response to a Surprise Event. In Transportation Research Record: Journal of the Transportation Research Board, No. 2185, Transportation Research Board of the National Academies, Washington, D.C., 2010, pp. 1-7.

5. Said, D., A. Abd El Halim, and Y. Hassan. Desirable Spiral Length Based on Driver Steering Behavior. In Transportation Research Record: Journal of the Transportation Research Board, No. 2092, Transportation Research Board of the National Academies, Washington, D.C., 2009, pp. 28-38.

6. Toffin, D., G. Reymond, A. Kemeny, and J. Droulez. Role of Steering Wheel Feedback on Driver Performance: Driving Simulator and Modeling Analysis. Vehicle System Dynamics Vol. 45, No. 4, 2007, pp. 375-388.

7. Bittner, A. C., Jr., O. Simsek, W.H. Levison, and J.L. Campbell. On-Road Versus Simulator Data in Driver Model Development: Driver Performance Model Experience. In Transportation Research Record: Journal of the Transportation Research Board, No. 1803, Transportation Research Board of the National Academies, Washington, D.C., 2002, pp. 38-44.

8. Odhams, A. M. C. Identification of Driver Steering and Speed Control. $\mathrm{PhD}$ dissertation. Queen's College, Cambridge University, Cambridge, United Kingdom, 2006.

9. Pradhan, A. K., D. L. Fisher, and A. Pollatsek. Risk Perception Training for Novice Drivers: Evaluating Duration of Effects of Training on a Driving Simulator. In Transportation Research Record: Journal of the Transportation Research Board, No. 1969, Transportation Research Board of the National Academies, Washington, D.C., 2006, pp. 58-64.

10. Allen, R. W., T. J. Rosenthal, and M. L. Cook. A Short History of Driving Simulation. In Handbook of Driving Simulation for Engineering, Medicine and Psychology (D. L. Fisher et al., eds.), CRC Press, Boca Raton, Fla., 2011, pp. 1-16.

11. Sony. Sony Playstation 4. 2014. http://ie.playstation.com/ps4. Accessed July 27, 2014.

12. Microsoft. Xbox One. 2014. http://www.xbox.com/en-IE/xbox-one /meet-xbox-one. Accessed July 27, 2014.

13. Mori, M., K. F. MacDorman, and N. Kageki. The Uncanny Valley [from the Field]. Robotics and Automation Magazine, Vol. 19, No. 2, 2012, pp. 98-100.

14. Ono, S., K. Ogawara, M. Kagesawa, H. Kawasaki, M. Onuki, J. Abeki, T. Yano, M. Nerio, K. Honda, and K. Ikeuchi. A Photo-Realistic Driving Simulation System for Mixed-Reality Traffic Experiment Space. IEEE Symposium on Intelligent Vehicles Symposium, Las Vegas, Nev., IEEE, New York, 2005, pp. 747-752.

15. Bredif, M. Image-Based Rendering of LOD1 3D City Models for Traffic-Augmented Immersive Street-View Navigation. ISPRS Annals of the Photogrammetry, Remote Sensing and Spatial Information Sciences, Volume II-3/W3, 2013, pp. 7-11.

16. de Ceunynck, T., C. Ariën, K. Brijs, T. Brijs, K. van Vlierden, J. Kuppens, M. van der Linden, and G. Wets. Proactive Evaluation of Traffic Signs Using a Traffic Sign Simulator. Presented at 93rd Annual Meeting of the Transportation Research Board, Washington, D.C., 2014.

17. Sato, R., S. Ono, H. Kawasaki, and K. Ikeuchi. Real-Time ImageBased Rendering System for Virtual City Based on Image Compression Technique and Eigen Texture Method. Proc., 19th International Conference on Pattern Recognition (ICPR). Tampa, Fla., 2008, pp. $1-4$.

18. Brogan, M., D. Kaneswaran, S. Commins, C. Markham, and C. Deegan. Automatic Generation and Population of a Graphics-Based Driving Simulator Use of Mobile Mapping Data for Behavioral Testing of 
Drivers. In Transportation Research Record: Journal of the Transportation Research Board, No. 2434, Transportation Research Board of the National Academies, Washington, D.C., 2014, pp. 95-102.

19. Allen, R. W., G. D. Park, and M. L. Cook. Simulator Fidelity and Validity in a Transfer-of-Training Context. In Transportation Research Record: Journal of the Transportation Research Board, No. 2185, Transportation Research Board of the National Academies, Washington, D.C., 2010, pp. 40-47.

20. Sahami S., and T. Sayed. Insight into Steering Adaptation Patterns in a Driving Simulator. In Transportation Research Record: Journal of the Transportation Research Board, No. 2185, Transportation Research Board of the National Academies, Washington, D.C., 2010, pp. 33-39.

21. Kaneswaran, D., M. Brogan, M. Mulcahy, S. Commins, C. Deegan, and C. Markham. Replicating Reality: Driver Assessment Using DualFidelity Simulator. Proc., 24th IET Irish Signals and Systems Conference, Letterkenny, Ireland, Institute of Engineering and Technology, Stevenage, United Kingdom, 2013, pp. 1-6.

22. Brogan, M., C. Markham, S. Commins, and C. Deegan. Steerable Video: Generating Video-Based Environments for Driving Simulation. Proc., Driving Simulation Conference (DSC), Paris, 2014.

23. Samsung Corp. Solid State Drives. 2014. http://www.samsung.com/ie /consumer/memory-cards-hdd-odd/ssd/. Accessed July 27, 2014.
24. Brogan, M., S. Haughey, S. McLoughlin, and C. Deegan. Low-Profile Mobile Mapping System. Proc., International Information Technology and Telecommunications Conference, Athlone, Ireland, 2013.

25. Point Grey Research. Bumblebee XB3. 2014. http://www.ptgrey.com /products/bbxb3/. Accessed July 27, 2014.

26. NovAtel. NovAtel: Pro-Pak V3. 2014. http://www.novatel.com/products /gnss-receivers/enclosures/propak-v3/. Accessed July 27, 2014.

27. Brogan, M., S. McLoughlin, and C. Deegan. Assessment of Stereo Camera Calibration Techniques for a Portable Mobile Mapping System. IET Journal of Computer Vision, Vol. 7, No. 3, 2013, pp. 209-217.

28. Hartley, R., and A. Zisserman. Multiple View Geometry in Computer Vision, 2nd ed. Cambridge University Press, Cambridge, United Kingdom, 2003.

29. Trucco, E., and A. Verri. Introductory Techniques for 3-D Computer Vision, 1st ed. Prentice Hall, Upper Saddle River, N.J., 1998.

30. OpenCV. OpenCV Image Processing Library. 2014. http://opencv.org/. Accessed July 27, 2014.

31. The FreeGLUT Project. Free OpenGL Utility Toolkit. 2014. http:// freeglut.sourceforge.net/. Accessed July 27, 2014.

The Standing Committee on Simulation and Measurement of Vehicle and Operator Performance peer-reviewed this paper. 